\title{
The activation state of macrophages alters their ability to suppress preadipocyte apoptosis
}

\author{
André S D Molgat, AnneMarie Gagnon, Charlie Foster and Alexander Sorisky \\ Chronic Disease Program, Ottawa Hospital Research Institute; and Departments of Medicine and of Biochemistry, Microbiology and Immunology, University of \\ Ottawa, General Campus, 501 Smyth Road, Ottawa, Ontario, Canada K1H 8L6 \\ (Correspondence should be addressed to A Sorisky; Email: asorisky@ohri.ca)
}

\begin{abstract}
Adipose tissue contains macrophages whose state of activation is regulated as obesity develops. Macrophagesecreted factors influence critical processes involved in adipose tissue homeostasis, including preadipocyte proliferation and differentiation into adipocytes. Macrophage-conditioned medium (MacCM) from J774A.1 macrophages protects 3T3-L1 preadipocytes from apoptosis through platelet-derived growth factor (PDGF) signaling. Here, we investigated the effect of macrophage activation on MacCM-dependent preadipocyte survival. MacCM was prepared following activation of either J774A.1 macrophages with lipopolysaccharide (LPS) or human primary monocytederived macrophages (MD-macrophages) with LPS or interleukin 4 (IL4). 3T3-L1 and human primary preadipocytes were induced to undergo apoptosis in MacCM, and apoptosis was quantified by cell enumeration or Hoechst nuclear staining. Preadipocyte PDGF signaling was assessed by immunoblot analysis of phosphorylated PDGF receptor,
\end{abstract}

Akt, and ERK1/2. Pro-inflammatory activation of J774A.1 macrophages with LPS inhibited the pro-survival activity of MacCM on 3T3-L1 preadipocytes, despite intact PDGF signaling. Upregulation of macrophage tumor necrosis factor a (TNF $\alpha)$ expression occurred in response to LPS, and TNF $\alpha$ was demonstrated to be responsible for the inability of LPS-J774A.1-MacCM to inhibit preadipocyte apoptosis. Furthermore, MacCM from human MD-macrophages (MD-MacCM) inhibited apoptosis of primary human preadipocytes. MD-MacCM from LPStreated macrophages, but not IL4-treated anti-inflammatory macrophages, was unable to protect human preadipocytes from cell death. In both murine cell lines and human primary cells, pro-inflammatory activation of macrophages inhibits their pro-survival activity, favoring preadipocyte death. These findings may be relevant to preadipocyte fate and adipose tissue remodeling in obesity.

Journal of Endocrinology (2012) 214, 21-29

\section{Introduction}

Obesity is a major risk factor for insulin resistance, type 2 diabetes, and cardiovascular disease. Adipose tissue expansion, due to chronic positive energy balance, occurs via coordinated increases in adipocyte size (hypertrophy) and number (hyperplasia). Hyperplastic obesity, associated with insulin sensitivity in humans and in animal models (Kim et al. 2007, Arner et al. 2010), depends on an adequate number of functional preadipocytes that can differentiate into adipocytes to meet the energy storage demand (adipogenesis). By contrast, hypertrophic obesity is linked to adipose tissue inflammation and insulin resistance (Heilbronn et al. 2004, Arner et al. 2010) and results from insufficient adipogenic capacity.

In addition to preadipocytes and adipocytes, adipose tissue contains macrophages (adipose tissue macrophages; ATMs). Their numbers and activation states vary with the extent of adiposity (Weisberg et al. 2003, Xu et al. 2003,

Lumeng et al. 2007). In the lean state, resident ATMs account for $\sim 10 \%$ of stromal cells and are in an M2 anti-inflammatory activation state. In obesity, due to infiltration and differentiation of circulating monocytes, ATMs comprise $\sim 50 \%$ of stromal cells, and many display a pro-inflammatory M1 activation state. These dynamic changes in macrophage activation alter adipose tissue function. Obese mice subjected to conditional ablation of CD11c-positive cells fail to accumulate M1 ATMs and are not susceptible to diet-induced insulin resistance (Patsouris et al. 2008). Mice with a macrophage-specific PPAR $\gamma$ deletion do not generate M2 macrophages and are predisposed to obesity-associated insulin resistance (Odegaard et al. 2007). Furthermore, depending on the macrophage model used, the antiadipogenic activity of macrophage-conditioned medium (MacCM) on cultured preadipocytes, reported by us and others (Constant et al. 2006, Lacasa et al. 2007, Ide et al. 2011), can be regulated by macrophage activation (Lumeng et al. 2008, Stienstra et al. 2008, Lu et al. 2010). 
Our previous investigations on macrophage-adipose cell interactions revealed that MacCM, collected under basal conditions, protects 3T3-L1 preadipocytes from apoptosis in a platelet-derived growth factor (PDGF)-dependent manner (Molgat et al. 2009, 2011). Pro-inflammatory environments, induced by a high-fat diet, or cytokines, such as interleukin 6 (IL6), IL1 $\beta$, and tumor necrosis factor a (TNF $\alpha)$, have been associated with adipose cell death, both in vitro and in vivo (Niesler et al. 2000, Tchkonia et al. 2005, Alkhouri et al. 2010, Keuper et al. 2011). Therefore, we have now investigated the effect of pro-inflammatory macrophage activation on the pro-survival activity of MacCM on preadipocytes, using mouse cell lines and human cells in primary culture.

\section{Materials and Methods}

\section{Preparation of MacCM}

J774A.1-MacCM was prepared from J774A.1 mouse macrophages (from ATCC, Manasas, VA, USA) that were grown in DMEM supplemented with 10\% FBS (Hyclone, South Logan, UT, USA), $100 \mathrm{U} / \mathrm{ml}$ penicillin, and $0.1 \mathrm{mg} / \mathrm{ml}$ streptomycin (Gibco). Confluent cells were placed in serum-free medium that was collected $24 \mathrm{~h}$ later. LPS-J774A.1-MacCM was prepared by treating confluent cells with $100 \mathrm{ng} / \mathrm{ml}$ lipopolysaccharide (LPS; SigmaAldrich) or vehicle (HBSS; Hank's buffered salt solution) for $0 \cdot 5-6 \mathrm{~h}$; cells were then placed in serum-free medium for $24 \mathrm{~h}$ of conditioning before collection. In some experiments, where indicated, J774A.1 macrophages in serum-free medium were treated with a lower dose of LPS $(10 \mathrm{ng} / \mathrm{ml})$ during the 24-h conditioning period. For these studies, to control any residual LPS bioactivity in the medium after collection, 3T3-L1 preadipocytes were treated with serumfree control medium supplemented with $10 \mathrm{ng} / \mathrm{ml}$ LPS. There was no effect of LPS control conditions on cell signaling or cell death responses. To neutralize TNF $\alpha$, LPS-J774A.1-MacCM or J774A.1-MacCM was incubated with either $2 \mu \mathrm{g} / \mathrm{ml}$ rat anti-mouse TNF $\alpha$ neutralizing antibody (R\&D Systems, Minneapolis, MN, USA) or control nonspecific rat $\operatorname{IgG}$ for $1 \mathrm{~h}$ before use.

To prepare MacCM from human monocyte-derived macrophages (MD-macrophages), peripheral blood mononuclear cells (PBMCs) were isolated from blood donated by six healthy volunteers (three males and three females; mean age $29 \cdot 7 \pm 7 \cdot 5$ years; approved by The Ottawa Hospital Research Ethics Board, No. 2008445-01H). Blood was centrifuged $(500 \mathrm{~g})$ with Ficoll-Hypaque technique (GE Healthcare, Uppsala, Sweden) and PBMCs were seeded at a density of $1 \times 10^{7}$ cells/well in 12-well culture dishes. Monocytes were allowed to adhere for $1 \mathrm{~h}$ in serum-free RPMI and subsequently differentiated into macrophages over either 7 or 14 days in RPMI supplemented with $10 \%$ FBS, $100 \mathrm{U} / \mathrm{ml}$ penicillin, and $0.1 \mathrm{mg} / \mathrm{ml}$ streptomycin (Daigneault et al.
2010, Mayi et al. 2010). Where indicated, macrophage differentiation was also induced in RPMI supplemented with $20 \% \mathrm{FBS}, 100 \mathrm{U} / \mathrm{ml}$ penicillin, $0 \cdot 1 \mathrm{mg} / \mathrm{ml}$ streptomycin, and $100 \mathrm{ng} / \mathrm{ml} \mathrm{M-CSF}$ for either 7 or 14 days (Martinez et al. 2006). Differentiated human macrophages were placed in serum-free medium and treated with either $100 \mathrm{ng} / \mathrm{ml}$ LPS (M1 activation) or $20 \mathrm{ng} / \mathrm{ml}$ human recombinant IL4 (M2 activation; R\&D Systems); the medium was collected 24-h later (monocyte-derived MacCM; MD-MacCM; Martinez et al. 2006). To control for any potential residual bioactivity in the medium during preadipocyte studies, unconditioned control medium supplemented with corresponding concentrations of LPS or IL4 was evaluated; no effect on preadipocyte responses was noted.

THP-1 monocytes (ATCC) were cultured as described (Constant et al. 2006). They were grown to a density of $1 \times 10^{6}$ cells $/ \mathrm{ml}$ and then treated with $100 \mathrm{nM} 12-O$-tetradecanoylphorbol-13-acetate (TPA) for $24 \mathrm{~h}$ to induce macrophage differentiation. The resulting macrophages were placed in serum-free medium without TPA, and the THP-1-MacCM was collected $24 \mathrm{~h}$ later.

MacCM, generated from each macrophage model, was centrifuged at $200 \mathrm{~g}$ for $5 \mathrm{~min}$, and the supernatant was stored at $-20{ }^{\circ} \mathrm{C}$ until use for preadipocyte studies.

\section{Isolation and culture of human preadipocytes}

Subcutaneous adipose tissue was obtained from healthy volunteers undergoing elective abdominal surgery (approved by The Ottawa Hospital Research Ethics Board, No. 1995023$01 \mathrm{H})$. Mean age was $52 \cdot 2 \pm 12$ years, and mean body mass index was $31 \cdot 2 \pm 11 \cdot 4 \mathrm{~kg} / \mathrm{m}^{2}$ (土s.D.). Preadipocytes were isolated as described (Artemenko et al. 2005). Adipose tissue was separated from the connective tissue and capillaries by dissection and then digested with collagenase CLS type I (600 U/g tissue; Worthington, Lakewood, NJ, USA). The digested tissue was subjected to progressive size filtration and centrifugation, followed by incubation in erythrocyte lysis buffer. Preadipocytes were seeded at a density of $3 \cdot 5 \times 10^{5}$ cells $/ 35 \mathrm{~mm}$ dish, cultured in DMEM supplemented with $10 \% \mathrm{FBS}, 100 \mathrm{U} / \mathrm{ml}$ penicillin, $0.1 \mathrm{mg} / \mathrm{ml}$ streptomycin, and $50 \mathrm{U} / \mathrm{ml}$ nystatin (Calbiochem; Merck KGaA, Darmstadt, Germany) and grown for 24-48 h until confluent.

\section{Preadipocyte signal transduction studies}

Low-passage 3T3-L1 mouse preadipocytes (ATCC) were grown in DMEM supplemented with $10 \%$ calf serum, $100 \mathrm{U} / \mathrm{ml}$ penicillin, and $0.1 \mathrm{mg} / \mathrm{ml}$ streptomycin (growth medium). Confluent 3T3-L1 preadipocytes, pretreated, where indicated, for $90 \mathrm{~min}$ with $10 \mu \mathrm{M}$ imatinib or vehicle (0.1\% DMSO), were placed in serum-free J774A.1-MacCM, LPS-J774A.1-MacCM, or control medium (supplemented with $10 \mathrm{ng} / \mathrm{ml} \mathrm{LPS}$, where indicated) for $15 \mathrm{~min}$. Preadipocytes were then processed for immunoblot analysis. 


\section{Immunoblot analysis}

Cells were lysed in $1 \times$ Laemmli buffer (Laemmli 1970) supplemented with 5\% $\beta$-mercaptoethanol, $1 \mathrm{mM}$ sodium orthovanadate, $5 \mathrm{mM}$ EGTA (pH 8.0), $50 \mathrm{mM}$ sodium fluoride, and $5 \mathrm{mM}$ sodium pyrophosphate. Protein was measured using the DC Protein Assay (Bio-Rad) with BSA as a standard. Equal amounts $(20-25 \mu \mathrm{g})$ of solubilized protein were resolved by SDS-PAGE and transferred to a nitrocellulose membrane. Membranes were incubated for $1 \mathrm{~h}$ in $5 \%$ skim milk or 3\% BSA to block nonspecific binding sites and then probed as indicated with the following primary antibodies directed against: PDGF receptor $\beta$ (PDGFR $\beta$; rabbit polyclonal, $1 \mu \mathrm{g} / \mathrm{ml}$; Santa Cruz Biotechnology, Santa Cruz, CA, USA), phosphotyrosine (PY20, mouse monoclonal, 1:1000; BD Biosciences, Mississauga, ON, Canada), ERK1/2 (rabbit polyclonal, 0.25 $\mu \mathrm{g} / \mathrm{ml}$; Upstate Biotechnology, Charlottesville, VA, USA), phospho-ERK1/2 (pERK1/2, Thr202/Tyr204, rabbit polyclonal, 1:2000), Akt (rabbit polyclonal, 1:1000), or phospho-Akt (pAkt, Ser473, rabbit polyclonal, 1:1000; all from Cell Signaling Technology, Beverly, MA, USA). After incubation with the appropriate HRP-conjugated secondary antibody, signal detection was performed using the Immobilon Western chemiluminescence HRP Substrate kit (Millipore, Billerica, MA, USA). Relative intensity of the bands was quantified using AlphaEaseFC software (Alpha Innotech, San Leandro, CA, USA) and expressed as integrated optical density (IOD) units.

\section{Cell death analysis}

For cell enumeration studies, confluent 3T3-L1 preadipocytes, pretreated, where indicated, for $90 \mathrm{~min}$ with $10 \mu \mathrm{M}$ imatinib or vehicle $(0 \cdot 1 \%$ DMSO), were placed in control medium (supplemented or not with $10 \%$ FBS, 1-100 ng/ml recombinant mouse TNF $\alpha$ (R\&D Systems), or $20 \mathrm{ng} / \mathrm{ml}$ recombinant human PDGF BB (Calbiochem)), serum-free J774A.1-MacCM, or serum-free LPS-J774A. 1-MacCM. After $6 \mathrm{~h}$, floating cells were removed, and adherent cells were trypsinized and stained with $0 \cdot 2 \%$ trypan blue dye. Viable cells (trypan blue exclusion) were counted in duplicate using a Neubauer hemocytometer. Each treatment was performed in duplicate.

For Hoechst staining, primary human preadipocytes grown on coverslips were placed in serum-free medium (control or MacCM) supplemented with either $10 \mu \mathrm{g} / \mathrm{ml}$ cycloheximide (CHX; Calbiochem), $10 \mathrm{ng} / \mathrm{ml}$ recombinant human TNF $\alpha$ (R\&D Systems), or both, for $6 \mathrm{~h}$ to induce apoptosis (Fischer-Posovszky et al. 2004, 2011, Tchoukalova et al. 2007). Cells were then fixed in $10 \%$ formaldehyde for $1 \mathrm{~h}$, followed by staining for $10 \mathrm{~min}$ with $1 \mu \mathrm{g} / \mathrm{ml}$ Hoechst 33342 in a humid chamber. Individual coverslips were mounted onto glass slides using Moviol. Cells were visualized and photographed $(400 \times$ magnification) with a Zeiss Axio Imager. M1 microscope equipped with an Axiocam $\mathrm{HRm}$ digital camera (Carl Zeiss, Toronto, Canada). Ten random fields, equivalent to a minimum of $\sim 200$ cells, were photographed for each of the three coverslips used per treatment. Percentage of apoptosis was calculated by dividing the total number of apoptotic nuclei by the total number of nuclei (counted by two independent observers) multiplied by 100 .

\section{RT-PCR}

J774A.1 macrophages were treated with $100 \mathrm{ng} / \mathrm{ml}$ LPS or vehicle $(0 \cdot 1 \% \mathrm{HBSS})$ for 2 or $6 \mathrm{~h}$. RNA, extracted with Qiazol (Qiagen), was processed and analyzed by RT-PCR as described (Ide et al. 2011). Data were analyzed using Light Cycler Software 3.0 (Roche) and are expressed as the fold increase in the relative quantification (RQ) of LPS-treated cells vs time-matched controls. TNF $\alpha$-specific primer pairs were as follows: forward, 5'-ACGGCATGGATCTCAAAGAC- $3^{\prime}$; reverse, 5'-AGATAGCAAATCGGCTGACG-3', with $18 \mathrm{~S}$ used as an internal control. The relative expression of PDGFA and -B mRNA was calculated by comparing the RQ values of each gene, normalized to 18 S, in untreated J774A.1 macrophages. Primer pairs for PDGF were as follows: PDGFA, forward 5'-GGAGCCAGGTGGACCCCACA-3' and reverse $5^{\prime}$-TGGCCACCTTGACACTGCGG-3'; and PDGFB, forward $5^{\prime}$-CCACTCCATCCGCTCCTTT- $3^{\prime}$ and reverse $5^{\prime}$-AAGTCCAGCTCAGCCCCAT-3'.

MD macrophages, treated with $100 \mathrm{ng} / \mathrm{ml} \mathrm{LPS} \mathrm{or} 20 \mathrm{ng} / \mathrm{ml}$ IL4 for $24 \mathrm{~h}$, were processed for RT-PCR analysis, as described earlier. $\mathrm{TNF} \boldsymbol{\alpha}$-specific primer pairs were as follows: forward, 5'-GCCCCCAGAGGGAAGAGTTCCC-3'; reverse, 5'-CAGCTCCACGCCATTGGCCA-3'. CD206-specific primer pairs were as follows: forward, $5^{\prime}$-GGAAGGCGGTGACCTCACAAGT-3'; reverse, 5'-TGCCCAGTACCCATCCTTGCCTT-3'. 18S was used as an internal control.

\section{ELISA}

PDGF BB content in basal J774A.1- and LPS-J774A.1-MacCM (prepared following treatment with $100 \mathrm{ng} / \mathrm{ml}$ LPS or vehicle for 2 or $6 \mathrm{~h}$ in serum-free medium) was assayed using the Quantikine mouse/rat PDGF BB ELISA (R\&D Systems). TNF $\alpha$ and IL1 $\beta$ were quantified in THP-1-MacCM using Quantikine human IL1 $\beta$ and human TNF $\alpha$ ELISA (R\&D Systems).

\section{Statistical analysis}

One-way ANOVA followed by the post hoc Newman-Keuls test was used to assess the differences between means using GraphPad InStat v3.05 (GraphPad Software, Inc., San Diego, CA, USA). For imatinib studies, two-way ANOVA followed by the post hoc Tukey's test was used. A value of $P<0 \cdot 05$ was considered significant. 


\section{Results}

Pro-inflammatory macrophage activation reduces the survival activity of J774A.1-MacCM

We previously reported that J774A.1-MacCM, in a PDGFdependent manner, inhibits apoptosis of serum-deprived 3T3-L1 preadipocytes, and this was documented by cell enumeration, Hoechst staining, and annexin $\mathrm{V}$ detection by flow cytometry (Molgat et al. 2009, 2011). We have now investigated, for the first time, the effect of pro-inflammatory macrophage activation, induced by LPS treatment, on the preadipocyte survival activity of MacCM. As described earlier, basal J774A.1-MacCM prevented serum withdrawal-induced death of 3T3-L1 preadipocytes, assessed by enumeration of viable cells (Fig. 1A). Addition of LPS to J774A. 1 macrophages for $6 \mathrm{~h}$ before the conditioning period inhibited survival activity of the MacCM by $50 \%$ (Fig. 1A). Shorter durations of LPS treatment were less effective.

To investigate the mechanism by which LPS reduces the pro-survival activity of MacCM, we measured the amount of PDGF released into J774A.1-MacCM, as its ability to
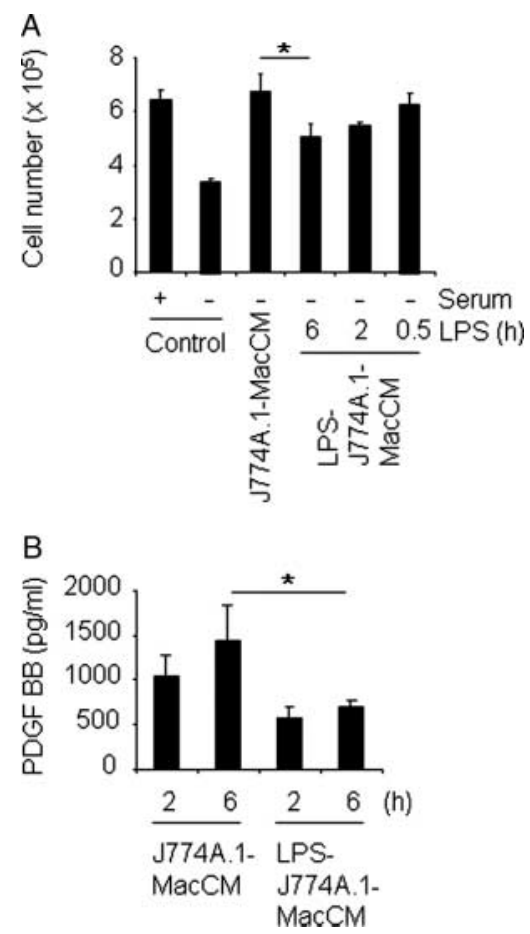

Figure 1 Pro-inflammatory macrophage activation, PDGF release, and survival activity of J774A.1-MacCM. (A) 3T3-L1 preadipocytes were incubated for $6 \mathrm{~h}$ in serum-free control medium, J774A. 1-MacCM, or LPS-J774A.1-MacCM generated by LPS pretreatment of macrophages for the times indicated. Viable adherent cells (trypan blue negative) were enumerated and expressed as mean cell number \pm S.E.M. $n=4, * P<0 \cdot 05$. (B) PDGF BB in J774A.1-MacCM or LPS-J774A.1-MacCM, treated with vehicle or LPS for the indicated period before the preparation of MacCM, was quantified by ELISA. Data are expressed in picogram per milliliter. $n=3, * P<0 \cdot 05$. suppress preadipocyte apoptosis is dependent on this growth factor (Molgat et al. 2009). RT-PCR comparative cycle number analysis indicated that PDGFB mRNA is 19-fold more abundant than PDGFA in unactivated macrophages (data not shown), which is in agreement with other observations (Nagaoka et al. 1992). J774A.1-MacCM contained 1034 or $1443 \mathrm{pg} / \mathrm{ml}$ of PDGF BB, following 2 or $6 \mathrm{~h}$ of vehicle treatment respectively (Fig. 1B). Treatment of macrophages with LPS for 2 or $6 \mathrm{~h}$ decreased the PDGF BB levels in MacCM by 44 or $52 \%$ respectively (Fig. 1B).

Given the lower level of PDGF protein released by the J774A.1 macrophages in response to LPS, we determined whether the reduced survival activity of LPS-J774A. 1-MacCM might be due to attenuated PDGF signaling in 3T3-L1 preadipocytes. We examined the phosphorylation responses of PDGFR, Akt, and ERK1/2, as we have previously reported that these pathways mediate the prosurvival effect of MacCM (Molgat et al. 2011). There was no difference in PDGFR or Akt phosphorylation in response to LPS-J774A.1-MacCM vs J774A.1-MacCM (Fig. 2A). Therefore, LPS-J774A.1-MacCM survival signaling in preadipocytes, as assessed by these two PDGFR-dependent parameters, was unaffected despite the LPS-associated reduction of PDGF protein levels in the MacCM (Fig. 1B).

ERK1/2 phosphorylation in 3T3-L1 preadipocytes treated with LPS-J774A.1-MacCM vs J774A.1-MacCM was actually enhanced (Fig. 2A). The augmented ERK1/2 signaling with LPS-J774A.1-MacCM was not altered by pretreating preadipocytes with imatinib (Fig. 2B), suggesting that PDGF was not responsible for the enhanced ERK1/2 signal.

LPS-induced macrophage TNF $\alpha$ production reduces the survival activity of J774A.1-MacCM

As PDGF survival signaling remained intact in LPS-J774A. 1-MacCM, we considered whether a pro-apoptotic factor was induced by LPS treatment. A 20 - or 40-fold increase in TNF $\alpha$ mRNA expression in J774A.1 macrophages occurred by 2 or $6 \mathrm{~h}$ of LPS treatment respectively (data not shown). TNF $\alpha$ promotes $3 \mathrm{~T} 3-\mathrm{L} 1$ preadipocyte apoptosis when added to a standard cell culture medium (Niesler et al. 2000). Therefore, we determined whether LPS-induced TNF $\alpha$ was responsible for the inhibition of MacCM pro-survival activity using an immunoneutralization strategy.

In this case, LPS-J774A.1-MacCM was collected after $24 \mathrm{~h}$ of $10 \mathrm{ng} / \mathrm{ml}$ LPS treatment and then added to 3T3-L1 preadipocytes. A similar increase in phosphorylation of PDGFR, Akt, and ERK1/2 occurred with this LPS protocol (Figs 2A vs 3A). LPS alone had no effect on 3T3-L1 preadipocyte signaling (Fig. 3A) or survival (data not shown). LPS-J774A.1-MacCM inhibited the pro-survival effect of unstimulated J774A.1-MacCM by 62\%, and TNF $\alpha$ immunoneutralization almost fully restored the pro-survival activity to $89 \%$ of the basal J774A.1-MacCM effect (Fig. 3B). The anti-TNF $\alpha$ antibody alone had no effect on cell death induced by serum withdrawal in the presence or absence of 

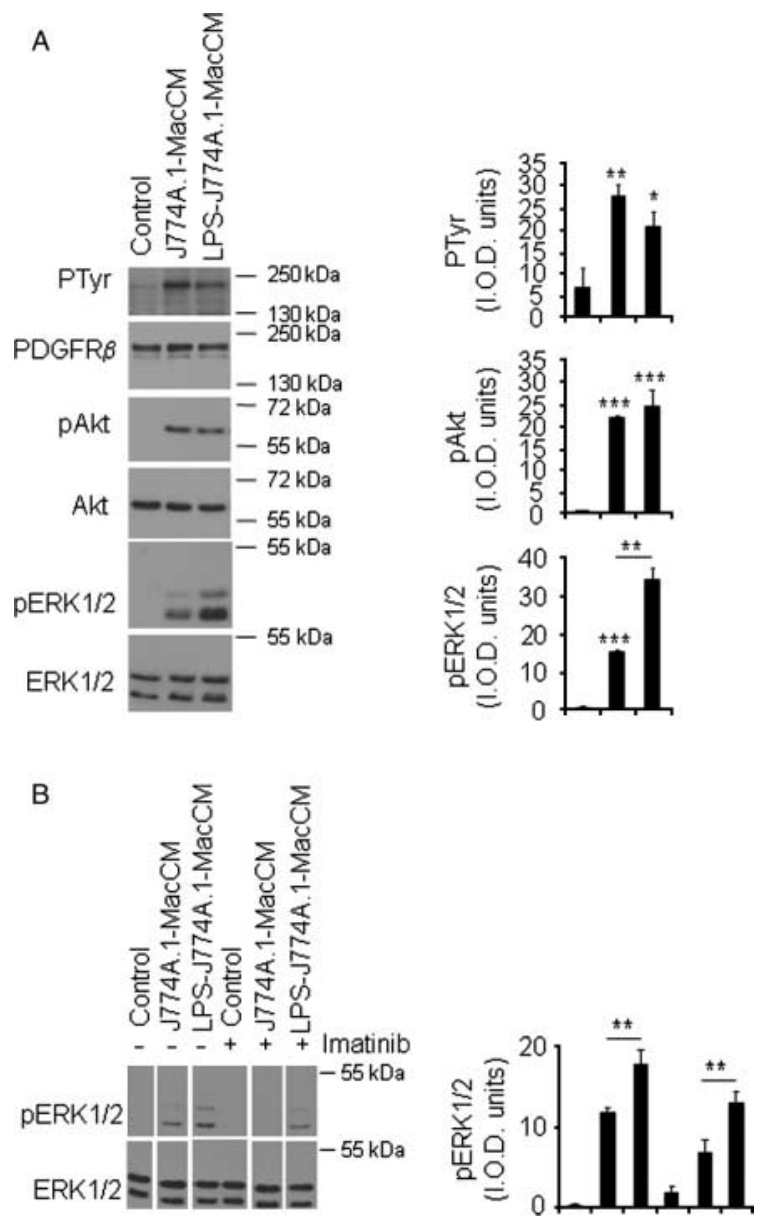

Figure 2 LPS-J774A.1-MacCM, PDGF signaling, and 3T3-L1 preadipocyte death. 3T3-L1 preadipocytes, pretreated or not with $10 \mu \mathrm{M}$ imatinib, as indicated, were incubated for $15 \mathrm{~min}$ in serumfree control medium, J774A.1-MacCM, or LPS-J774A.

1-MacCM (generated by LPS pretreatment of macrophages for $6 \mathrm{~h}$ ). Whole cell lysates were immunoblotted with the indicated antibodies. $n=3$ (A), $n=4(\mathrm{~B}), * P<0 \cdot 05, * * P<0 \cdot 01, * * * P<0 \cdot 001$ vs control medium, or indicated pairs.

LPS (data not shown). When TNF $\alpha$ was added to basal J774A.1-MacCM (no LPS treatment), it inhibited the ability of MacCM to suppress preadipocyte cell death (Fig. 3C). Therefore, TNF $\alpha$ appears to be a critical component of the LPS-J774A.1-MacCM that overcomes the pro-survival effect of the J774A.1 macrophage-derived PDGF on 3T3-L1 preadipocytes. TNF $\alpha$, when added to J774A.1-MacCM, is also sufficient to mimic the negative effect of LPS-J774A.1-MacCM on 3T3-L1 preadipocyte survival.

\section{MD-MacCM promotes the survival of human primary preadipocytes}

We evaluated whether the MacCM pro-survival effect observed with the J774A.1 and 3T3-L1 cell line models extends to interactions between human MD-macrophages and human stromal-vascular preadipocytes. However, compared with 3T3-L1 preadipocytes, human preadipocytes are much less susceptible to apoptosis induced solely by serum withdrawal (Papineau et al. 2003). To induce adequate cell death, it was necessary to supplement the serum-free medium with CHX and TNF $\alpha$, apoptotic inducers used together by others (Fischer-Posovszky et al. 2004, 2011, Tchoukalova et al. 2007). An approximate 20 -fold induction of human preadipocyte apoptosis occurred with this combination (Fig. 4A).

To investigate whether MacCM from primary macrophages would reduce preadipocyte apoptosis, we differentiated human blood monocytes into macrophages over 7 or 14 days in $10 \%$ FBS and then conditioned in serum-free medium for $24 \mathrm{~h}$. This MD-MacCM suppressed human preadipocyte apoptosis by either 41 or $55 \%$ respectively (Fig. 4B). A similar 36 or $55 \%$ suppression of apoptosis was also observed with MD-MacCM generated from macrophages that were differentiated as earlier but supplemented with M-CSF (Fig. 4B). Therefore, we have established for the first time that the pro-survival effect of macrophages on preadipocytes that occurs in murine cell lines is also valid for primary human cells.

We examined whether the pro-survival effect on preadipocytes might be altered by macrophages that were in a pro-inflammatory M1 vs anti-inflammatory M2 macrophage activation state. Either $100 \mathrm{ng} / \mathrm{ml}$ LPS (M1) or $20 \mathrm{ng} / \mathrm{ml}$ IL4 (M2) was added to serum-free medium during the $24 \mathrm{~h}$ conditioning period, resulting in a fivefold increase in TNF $\alpha$ mRNA $(P<0 \cdot 05)$ or a fourfold increase in CD206 mRNA $(P<0 \cdot 01)$ respectively. LPS-MD-MacCM not only lost its pro-survival effect but also exacerbated preadipocyte apoptosis by 1.4-fold (Fig. 4C). By contrast, IL4-MD-MacCM and basal MD-MacCM had similar pro-survival activities toward human preadipocytes (Fig. 4D). Neither LPS nor IL4, when added alone to control medium, affected human preadipocyte viability.

\section{Discussion}

Proliferation and differentiation of preadipocytes are influenced by macrophage-secreted factors (Constant et al. 2006, Lacasa et al. 2007, Maumus et al. 2008, Ide et al. 2011). We have studied another cell fate, survival, and have previously reported that MacCM from J774A.1 macrophages protects 3T3-L1 preadipocytes from apoptosis in a PDGF-dependent manner, measured by cell enumeration, Hoechst staining, and annexin V detection by flow cytometry (Molgat et al. 2009, 2011). Here, we report that pro-inflammatory macrophage activation reduces the pro-survival activity of J774A. 1-MacCM for 3T3-L1 preadipocytes. Furthermore, we have now demonstrated that human primary MD-macrophages exert a similar anti-apoptotic effect on human primary preadipocytes that is also dependent on the state of macrophage activation. 
A
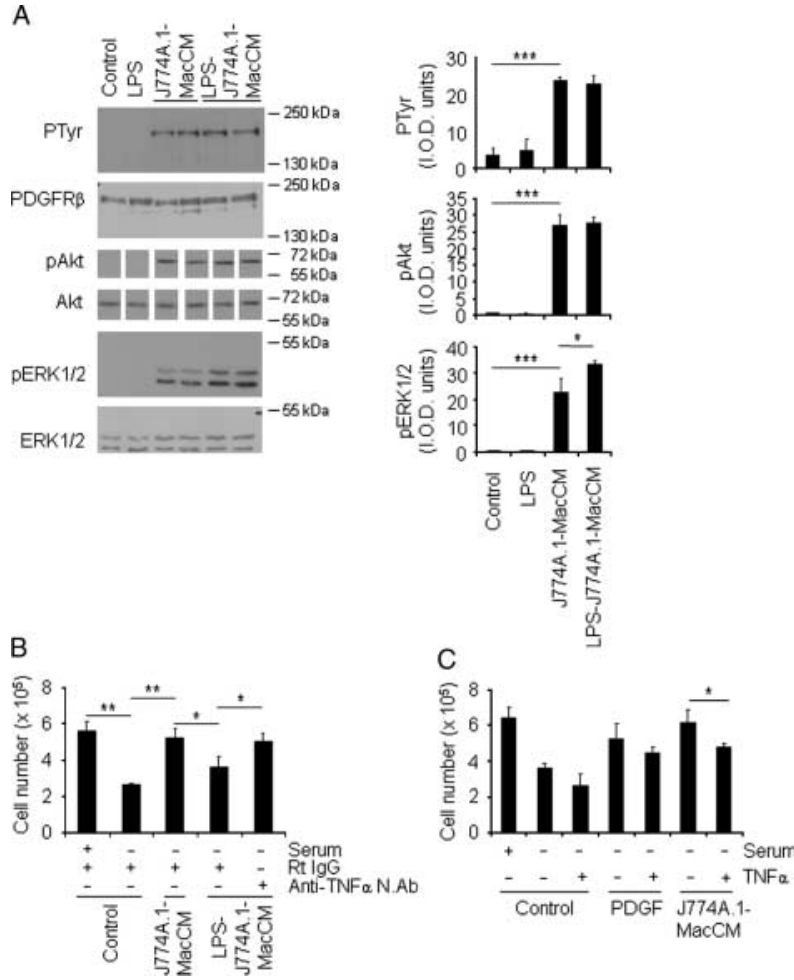

Figure 3 LPS-induced macrophage TNF $\alpha$ and survival activity of MacCM. (A) 3T3-L1 preadipocytes were incubated for $15 \mathrm{~min}$ in serum-free control medium, LPS control medium, J774A.1-MacCM, or LPS-J774A.1-MacCM, prepared following $24 \mathrm{~h}$ incubation with $10 \mathrm{ng} / \mathrm{ml}$ LPS. Whole cell lysates were immunoblotted with the indicated antibodies. $n=3, * P<0 \cdot 05, * * * P<0 \cdot 001$. (B) $3 \mathrm{~T} 3-\mathrm{L} 1$ preadipocytes were incubated for $6 \mathrm{~h}$ in serum-free control medium, J774A.1-MacCM, or LPS-J774A.1-MacCM, following preincubation of the media with anti-TNF $\alpha$ neutralizing antibody or control nonspecific rat IgG. Viable adherent cells (trypan blue negative) were enumerated and data expressed as mean cell number \pm s.E.M. $n=3,{ }^{*} P<0 \cdot 05, * * P<0 \cdot 01$. (C) $3 \mathrm{~T} 3-\mathrm{L} 1$ preadipocytes were incubated for $6 \mathrm{~h}$ with serum-free control medium, 20 ng/ml PDGF BB, or J774A.1-MacCM, each supplemented or not with $10 \mathrm{ng} / \mathrm{ml}$ TNF $\alpha$. Viable adherent cells (trypan blue negative) were enumerated and data expressed as mean cell number \pm S.E.M. $n=3, * P<0 \cdot 05$.

MacCM from LPS-treated vs basal J774A.1 macrophages displayed a reduced preadipocyte pro-survival activity. This occurred despite the ability of LPS-J774A.1-MacCM to fully activate PDGFR signaling pathways. The steady level of PDGF signaling, despite the reduced levels of PDGF, is consistent with the notion that PDGF levels produced by LPS-treated J774A.1 macrophages exceed a threshold concentration of PDGF sufficient for complete receptor activation and downstream signaling in 3T3-L1 cells. The pro-inflammatory state was associated with a significant upregulation of TNF $\alpha$. This cytokine is expressed at very low levels in J774A.1 cells under basal conditions and is rapidly upregulated by LPS to reach concentrations in the nanogram per milliliter range (Telepnev et al. 2003,
Jozefowski et al. 2010). Exogenous addition of TNF $\alpha$ in this concentration range was sufficient to inhibit the antiapoptotic effect of J774A.1-MacCM on 3T3-L1 preadipocytes. Immunoneutralization of $\mathrm{TNF} \alpha$ in the MacCM demonstrated that this cytokine is responsible for the inhibition of the pro-survival activity. Our data indicate that TNF $\alpha$ interferes with the pro-survival effect of PDGF in the context of murine macrophage and preadipocyte models. This action on preadipocyte fate may potentially represent another mechanism by which TNF $\alpha$, emanating from either activated macrophages or inflamed hypertrophied adipocytes in vivo, can act as a local adipostat to limit adipose tissue expansion (Skolnik \& Marcusohn 1996, Zhang et al. 2001).

Until now, our investigations on the pro-survival effect of MacCM on preadipocytes were based on studies using mouse 3T3-L1 preadipocytes and mouse macrophage cell models (Molgat et al. 2009, 2011). Here, we have taken the important step to demonstrate that human MD-MacCM also prevents apoptosis of human preadipocytes. Compared with 3T3-L1 preadipocytes, human preadipocytes are relatively resistant to apoptosis induced by serum deprivation (Papineau et al. 2003). Therefore, we used an apoptotic trigger that consists of serum deprivation in the presence of CHX and TNF $\alpha$ (Fischer-Posovszky et al. 2004, 2011, Tchoukalova et al. 2007). TNF $\alpha$ activates the extrinsic apoptosis pathway through caspase- 8 activation vs the intrinsic mitochondriadependent apoptotic pathway induced by serum deprivation. CHX treatment inhibits pro-survival protein synthesis and has been shown to promote the association of caspase- 8 with the TNF receptor, which enhances the death-inducing activity of TNF $\alpha$ (Chau et al. 2011). In preadipocytes, CHX has been shown to selectively downregulate FLICE-like inhibitory protein, enhancing the sensitivity of these cells to death ligand-dependent apoptosis (Fischer-Posovszky et al. 2011). Our finding that MD-MacCM is capable of preventing $\mathrm{CHX} / \mathrm{TNF} \alpha-$ and serum deprivation-dependent preadipocyte apoptosis suggests that it impedes intrinsic and extrinsic apoptotic pathways.

The significant suppression of apoptosis of human preadipocytes by basal MD-MacCM was absent in LPS-MD-MacCM. Therefore, as for J774A.1 macrophages, pro-inflammatory M1 activation of MD-macrophages interferes with the pro-survival effect of MD-MacCM. Moreover, in the case of LPS-MD-MacCM, an augmentation of human preadipocyte apoptosis was observed, possibly arising from differences in the primary human macrophage model, from the higher LPS concentration used, or from the exogenous TNF $\alpha$ already present in the apoptosis-induction medium. The effect of an anti-inflammatory M2 stimulus on MD macrophages was studied using IL4 (Gordon \& Martinez 2010). IL4-activated MD-MacCM or basal untreated MD-MacCM each exerted similar anti-apoptotic effects on human preadipocytes. Therefore, the influence of MD-MacCM on human preadipocyte apoptosis appears to be activation dependent, as observed with J774A.1-MacCM. 
A

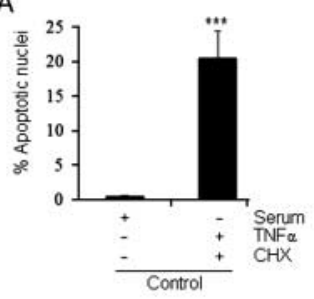

C

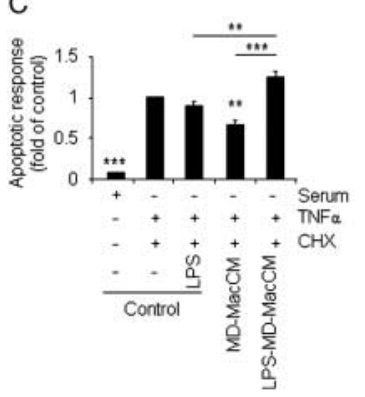

B
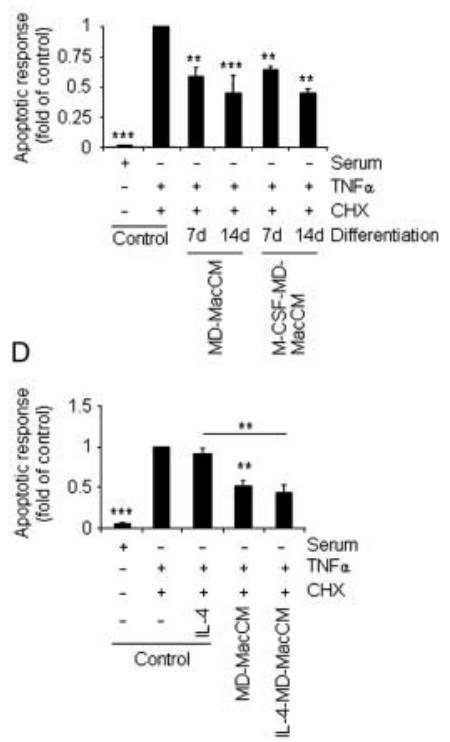

Figure 4 MD-MacCM survival activity and human preadipocytes. (A) Human primary preadipocytes were incubated for $6 \mathrm{~h}$ with serum-containing or serum-free medium supplemented with $10 \mu \mathrm{g} / \mathrm{ml} \mathrm{CHX}$ and $10 \mathrm{ng} / \mathrm{ml} \mathrm{TNF} \alpha$, as indicated. Cell death was assessed by quantification of Hoechst-stained apoptotic nuclei. $n=9,{ }^{* * *} P<0 \cdot 001$. (B) Human primary preadipocytes were induced to undergo apoptosis as described in $(A)$, in the presence of control medium or MD-MacCM, prepared following differentiation of human blood monocytes for 7 or 14 days in serum-containing medium supplemented or not with M-CSF. Cell death was assessed as in (A). Data are expressed as the fold of the control apoptotic response \pm s.E.M. $n=4,{ }^{* *} P<0 \cdot 01,{ }^{* * *} P<0 \cdot 001$ vs serum-free control. ( $C$ and $D)$ Human primary preadipocytes were induced to undergo apoptosis as described in (A) in the presence of MD-MacCM that was either unactivated or activated with either LPS (C) or IL4 (D). Cell death was assessed as in (A). Data are expressed as the fold of the control apoptotic response \pm s.E.M. $n=3,{ }^{* *} P<0.01$ and ${ }^{* * *} P<0.001$ vs serum-free control, or indicated pairs.

Future investigations will be necessary to elucidate the prosurvival mechanism in more detail. As an initial step, we have determined that Akt and ERK1/2 activation occurs in human preadipocytes in response to MD-MacCM $(n=3$, data not shown). These signaling responses were unaffected by the activation state of the macrophages, consistent with what we observed for the murine macrophage-preadipocyte studies. It will be important to directly assess whether Akt and ERK1/2 are implicated in MD-MacCM-dependent human preadipocyte survival and to identify the pro-survival factor(s) secreted by human macrophages in the basal or IL4-treated state. Furthermore, the basis of the blockade of the pro-survival effect of LPS-MD-MacCM on human preadipocytes remains to be determined. As noted, in contrast to the 3T3-L1 model in which apoptosis was triggered with serum deprivation alone, a more complex apoptotic induction was required for the human preadipocytes. The ability of basal and IL4-treated human MD-MacCM to protect human preadipocytes from death, despite the presence of exogenous TNF $\alpha$ in the treatment medium, will require further investigation. For example, human MD-macrophages may secrete a variety of pro-survival factors.

In contrast to the anti-apoptotic effect of MD-MacCM that we observed with primary human preadipocytes, a proapoptotic effect of THP-1-MacCM associated with the Simpson-Golabi-Behmel syndrome human preadipocyte model has been described (Keuper et al. 2011). Several reasons might account for this difference. The differentiation of THP-1 macrophages with $200 \mathrm{nM}$ phorbol myristate acetate by that group occurred over $48 \mathrm{~h}$, and elevated mRNA expression of IL6, IL1 $\beta$, and TNF $\alpha$ was noted, indicating a pro-inflammatory state. We have generated THP-1-MacCM derived from THP-1 macrophages that were differentiated with $100 \mathrm{nM}$ TPA over $24 \mathrm{~h}$ and observed a 30\% reduction in human preadipocyte apoptosis. With respect to cytokines released, our THP-1-MacCM contained similar levels of IL1 $\beta(447 \pm 59 \mathrm{pg} / \mathrm{ml}, n=3)$, but levels of $\mathrm{TNF} \alpha(21 \pm 10 \mathrm{pg} / \mathrm{ml}, n=3)$ were much lower compared with the nanogram per milliliter levels observed by Keuper et al. We do note that different commercial ELISA systems were used and perhaps that may have contributed in part to the variation in $T N F \boldsymbol{\alpha}$ values. In addition, compared with the primary human preadipocytes used in our studies, SimpsonGolabi-Behmel syndrome preadipocytes have a greater adipogenic capacity and higher sensitivity to apoptosis (Fischer-Posovszky et al. 2004).

Macrophage activation states could potentially alter the balance between adipocyte hypertrophy and hyperplasia through the control of preadipocyte survival and/or cell number. Hypertrophic adipose tissue growth is associated with insulin resistance and adipose tissue inflammation, whereas hyperplastic growth maintains adipose tissue insulin sensitivity. Our novel data, demonstrating that pro-inflammatory activation interferes with the anti-apoptotic activity of macrophages, are derived from cell culture models. Future studies are needed to address whether preadipocyte survival is regulated by ATMs in vivo and whether the pro-inflammatory activation of ATMs in the pathological state of obesity regulates preadipocyte viability.

\section{Declaration of interest}

The authors declare that there is no conflict of interest that could be perceived as prejudicing the impartiality of the research reported.

\section{Funding}

This work was supported by Heart and Stroke Foundation of Canada (Ontario) grant-in-aid NA6634 to A S. A S D M is the recipient of the Alexander Graham Bell Canada Doctoral Research Scholarship from the National Sciences and Engineering Research Council of Canada. 


\section{Acknowledgements}

The authors thank the patients and the surgeons of The Ottawa Hospital for their participation in their study.

\section{References}

Alkhouri N, Gornicka A, Berk MP, Thapaliya S, Dixon LJ, Kashyap S, Schauer PR \& Feldstein AE 2010 Adipocyte apoptosis, a link between obesity, insulin resistance, and hepatic steatosis. Journal of Biological Chemistry 285 3428-3438. (doi:10.1074/jbc.M109.074252)

Arner E, Westermark PO, Spalding KL, Britton T, Ryden M, Frisen J, Bernard S \& Arner P 2010 Adipocyte turnover: relevance to human adipose tissue morphology. Diabetes 59 105-109. (doi:10.2337/db09-0942)

Artemenko Y, Gagnon A, Aubin D \& Sorisky A 2005 Anti-adipogenic effect of PDGF is reversed by PKC inhibition. Journal of Cellular Physiology 204 646-653. (doi:10.1002/jcp.20314)

Chau H, Mirtsos C \& Huang HL 2011 Regulation of death complexes formation in tumor necrosis factor receptor signaling. Experimental Cell Research 317 1841-1850. (doi:10.1016/j.yexcr.2011.05.015)

Constant VA, Gagnon A, Landry A \& Sorisky A 2006 Macrophageconditioned medium inhibits the differentiation of 3T3-L1 and human abdominal preadipocytes. Diabetologia 49 1402-1411. (doi:10.1007/ s00125-006-0253-0)

Daigneault M, Preston JA, Marriott HM, Whyte MK \& Dockrell DH 2010 The identification of markers of macrophage differentiation in PMAstimulated THP-1 cells and monocyte-derived macrophages. PLoS ONE 5 e8668. (doi:10.1371/journal.pone.0008668)

Fischer-Posovszky P, Tornqvist H, Debatin KM \& Wabitsch M 2004 Inhibition of death-receptor mediated apoptosis in human adipocytes by the insulin-like growth factor I (IGF-I)/IGF-I receptor autocrine circuit. Endocrinology 145 1849-1859. (doi:10.1210/en.2003-0985)

Fischer-Posovszky P, Keuper M, Nagel S, Hesse D, Schurmann A, Debatin KM, Strauss G \& Wabitsch M 2011 Downregulation of FLIP by cycloheximide sensitizes human fat cells to CD95-induced apoptosis. Experimental Cell Research 317 2200-2209. (doi:10.1016/j.yexcr. 2011.06.016)

Gordon S \& Martinez FO 2010 Alternative activation of macrophages: mechanism and functions. Immunity 32 593-604. (doi:10.1016/j.immuni. 2010.05.007)

Heilbronn L, Smith SR \& Ravussin E 2004 Failure of fat cell proliferation, mitochondrial function and fat oxidation results in ectopic fat storage, insulin resistance and type II diabetes mellitus. International Journal of Obesity and Related Metabolic Disorders 28 (Suppl 4) S12-S21. (doi:10.1038/sj.ijo. 0802853)

Ide J, Gagnon A, Molgat AS, Landry A, Foster C \& Sorisky A 2011 Macrophage-conditioned medium inhibits the activation of cyclindependent kinase 2 by adipogenic inducers in 3T3-L1 preadipocytes. Journal of Cellular Physiology 226 2297-2306. (doi:10.1002/jcp.22566)

Jozefowski S, Czerkies M, Lukasik A, Bielawska A, Bielawski J, Kwiatkowska K \& Sobota A 2010 Ceramide and ceramide 1-phosphate are negative regulators of TNF- $\alpha$ production induced by lipopolysaccharide. Journal of Immunology 185 6960-6973. (doi:10.4049/ jimmunol.0902926)

Keuper M, Bluher M, Schon MR, Moller P, Dzyakanchuk A, Amrein K, Debatin KM, Wabitsch M \& Fischer-Posovszky P 2011 An inflammatory micro-environment promotes human adipocyte apoptosis. Molecular and Cellular Endocrinology 339 105-113. (doi:10.1016/j.mce.2011. 04.004)

Kim JY, van de Wall E, Laplante M, Azzara A, Trujillo ME, Hofmann SM, Schraw T, Durand JL, Li H, Li G et al. 2007 Obesity-associated improvements in metabolic profile through expansion of adipose tissue. Journal of Clinical Investigation 117 2621-2637. (doi:10.1172/JCI31021)
Lacasa D, Taleb S, Keophiphath M, Miranville A \& Clement K 2007 Macrophage-secreted factors impair human adipogenesis: involvement of proinflammatory state in preadipocytes. Endocrinology 148 868-877. (doi:10.1210/en.2006-0687)

Laemmli UK 1970 Cleavage of structural proteins during the assembly of the head of bacteriophage T4. Nature 227 680-685. (doi:10.1038/ 227680a0)

Lu C, Kumar PA, Fan Y, Sperling MA \& Menon RK 2010 A novel effect of growth hormone on macrophage modulates macrophage-dependent adipocyte differentiation. Endocrinology 151 2189-2199. (doi:10.1210/en. 2009-1194)

Lumeng CN, Bodzin JL \& Saltiel AR 2007 Obesity induces a phenotypic switch in adipose tissue macrophage polarization. Journal of Clinical Investigation 117 175-184. (doi:10.1172/JCI29881)

Lumeng CN, DelProposto JB, Westcott DJ \& Saltiel AR 2008 Phenotypic switching of adipose tissue macrophages with obesity is generated by spatiotemporal differences in macrophage subtypes. Diabetes 57 3239-3246. (doi:10.2337/db08-0872)

Martinez FO, Gordon S, Locati M \& Mantovani A 2006 Transcriptional profiling of the human monocyte-to-macrophage differentiation and polarization: new molecules and patterns of gene expression. Journal of Immunology 177 7303-7311.

Maumus M, Sengenes C, Decaunes P, Zakaroff-Girard A, Bourlier V, Lafontan M, Galitzky J \& Bouloumie A 2008 Evidence of in situ proliferation of adult adipose tissue-derived progenitor cells: influence of fat mass microenvironment and growth. Journal of Clinical Endocrinology and Metabolism 93 4098-4106. (doi:10.1210/jc.2008-0044)

Mayi TH, Duhem C, Copin C, Bouhlel MA, Rigamonti E, Pattou F, Staels B \& Chinetti-Gbaguidi G 2010 Visfatin is induced by peroxisome proliferator-activated receptor gamma in human macrophages. FEBS Journal 277 3308-3320. (doi:10.1111/j.1742-4658.2010.07729.x)

Molgat AS, Gagnon A \& Sorisky A 2009 Preadipocyte apoptosis is prevented by macrophage-conditioned medium in a PDGF-dependent manner. American Journal of Physiology. Cell Physiology 296 C757-C765. (doi:10.1152/ajpcell.00617.2008)

Molgat AS, Gagnon A \& Sorisky A 2011 Macrophage-induced preadipocyte survival depends on signaling through Akt, ERK1/2, and reactive oxygen species. Experimental Cell Research 317 521-530. (doi:10.1016/j.yexcr.2010. 10.024)

Nagaoka I, Honma S, Someya A, Iwabuchi K \& Yamashita T 1992 Differential expression of the platelet-derived growth factor-A and -B genes during maturation of monocytes to macrophages. Comparative Biochemistry and Physiology, Part B 103 349-356. (doi:10.1016/03050491(92)90303-9)

Niesler CU, Urso B, Prins JB \& Siddle K 2000 IGF-I inhibits apoptosis induced by serum withdrawal, but potentiates TNF- $\alpha$-induced apoptosis, in 3T3-L1 preadipocytes. Journal of Endocrinology 167 165-174. (doi:10.1677/joe.0.1670165)

Odegaard JI, Ricardo-Gonzalez RR, Goforth MH, Morel CR, Subramanian V, Mukundan L, Red Eagle A, Vats D, Brombacher F, Ferrante AW et al. 2007 Macrophage-specific PPARgamma controls alternative activation and improves insulin resistance. Nature $\mathbf{4 4 7}$ 1116-1120. (doi:10.1038/nature05894)

Papineau D, Gagnon A \& Sorisky A 2003 Apoptosis of human abdominal preadipocytes before and after differentiation into adipocytes in culture. Metabolism 52 987-992. (doi:10.1016/S0026-0495 (03)00165-3)

Patsouris D, Li PP, Thapar D, Chapman J, Olefsky JM \& Neels JG 2008 Ablation of CD11c-positive cells normalizes insulin sensitivity in obese insulin resistant animals. Cell Metabolism 8 301-309. (doi:10.1016/j.cmet. 2008.08.015)

Skolnik EY \& Marcusohn J 1996 Inhibition of insulin receptor signaling by TNF: potential role in obesity and non-insulin-dependent diabetes mellitus. Cytokine \& Growth Factor Reviews 7 161-173. (doi:10.1016/13596101(96)00021-4)

Stienstra R, Duval C, Keshtkar S, van der Laak J, Kersten S, Kersten S \& Muller M 2008 Peroxisome proliferator-activated receptor gamma 
activation promotes infiltration of alternatively activated macrophages into adipose tissue. Journal of Biological Chemistry 283 22620-22627. (doi:10. 1074/jbc.M710314200)

Tchkonia T, Tchoukalova YD, Giorgadze N, Pirtskhalava T, Karagiannides I, Forse RA, Koo A, Stevenson M, Chinnappan D, Cartwright A et al. 2005 Abundance of two human preadipocyte subtypes with distinct capacities for replication, adipogenesis, and apoptosis varies among fat depots. American Journal of Physiology. Endocrinology and Metabolism 288 E267-E277. (doi:10.1152/ajpendo.00265.2004)

Tchoukalova Y, Koutsari C \& Jensen M 2007 Committed subcutaneous preadipocytes are reduced in human obesity. Diabetologia 50 151-157. (doi:10.1007/s00125-006-0496-9)

Telepnev M, Golovliov I, Grundstrom T, Tarnvik A \& Sjostedt A 2003 Francisella tularensis inhibits Toll-like receptor-mediated activation of intracellular signalling and secretion of TNF- $\alpha$ and IL- 1 from murine macrophages. Cellular Microbiology 5 41-51. (doi:10.1046/j.1462-5822. 2003.00251.x)
Weisberg SP, McCann D, Desai M, Rosenbaum M, Leibel RL \& Ferrante AW Jr 2003 Obesity is associated with macrophage accumulation in adipose tissue. Journal of Clinical Investigation 112 1796-1808. (doi:10.1172/JCI200319246)

Xu H, Barnes GT, Yang Q, Tan G, Yang D, Chou CJ, Sole J, Nichols A, Ross JS, Tartaglia LA et al. 2003 Chronic inflammation in fat plays a crucial role in the development of obesity-related insulin resistance. Journal of Clinical Investigation 112 1821-1830. (doi:10.1172/JCI200319451)

Zhang HH, Kumar S, Barnett AH \& Eggo MC 2001 Dexamethasone inhibits tumor necrosis factor-alpha-induced apoptosis and interleukin-1 beta release in human subcutaneous adipocytes and preadipocytes. Journal of Clinical Endocrinology and Metabolism 86 2817-2825. (doi:10.1210/jc.86.6.2817)

Received in final form 1 May 2012

Accepted 3 May 2012

Made available online as an Accepted Preprint 3 May 2012 